WADC TECHNICAL REPORT 57-457

ASTIA DOCUMENT No. AD 130942

\title{
PSYCHOLOGICAL STRESS AS A THEORETICAL CONCEPT
}

W. DEAN CHILES

AERO MEDICAL LABORATORY

JÚLY 1957

Project No. 7193

WRIGHT AIR DEVELOPMENT CENTER

AIR RESEARCH AND DEVELOPMENT COMMAND

UNITED STATES AIR FORCE

WRIGHT-PATTERSON AIR FORCE BASE, OHIO

Carpenter Litho \& Prtg. Co., Springfield, 0.
500 - January 1958 